\title{
Research on The Model of Distributed Generation Under The DQ Axis Chenghui Lin ${ }^{1, a}$, Genjun Chen ${ }^{2, a}$,Yong Xiao ${ }^{1, a}$, Wei Gu ${ }^{1, a}$, Xiankui Wen ${ }^{1, a}$, Qiufeng Long ${ }^{1, a}$ \\ ${ }^{1}$ Electric Power Research Institute of State Grid GuiZhou Electric Power Company Guiyang, China \\ ${ }^{2}$ NARI Technology Development Co. Ltd. Nanjing, China \\ a158269975@qq.com
}

Keywords: distribution network; distributed generation; model; dq axis

\begin{abstract}
In order to conform to the development trend, distributed generation came into being.Distributed generation accesses to distribution network, which has increased the burden of the distribution network. One the one hand, distributed generation's accessing position is changeable and accessing capacity is increasing. On the other hand, as a dynamic component, distributed generation has its controlling problem and also brings stability problem to the main network. Starting from the modeling of distributed generation, this paper established distributed generation's circuit and controller model under the dq axis. These models are symmetric but the voltage of the distribution network is asymmetric. So a method was put forward solving the contradiction between the symmetric distributed generation model and the asymmetric voltage of the distribution network.
\end{abstract}

\section{Introduction}

In order to reduce the pressure on the environment and energy, distributed generation is promoted. Distributed generation (DG) develops rapidly for its advantages, such as a small investment, being friendly to environment, reliable supply and flexible power generation[1-3]. Distribution network (DN) is the access network of the distribution generation, and will inevitably be influenced by DG. DN's power quality and local voltage stability will be affected with the growing number and capacity of the DG, and the voltage stability problem belongs to the category of the transient research[4-7]. The traditional DN has almost no dynamic element, so the transient research attracts little attention. The access of the DG makes the transient research of the DN come into being. However, the conventional transient simulation containing DG isn't deeply involved in the problem of asymmetric network, and simulation is based on some commercial software such as matlab, PASCAD and DIgSILENT, which leads to the lack of knowledge of the specific process and the key link of the time domain simulation[8-13].

This paper mainly finishes the model of the elements of the distribution network containing the distributed generation, and the electromechanical transient simulation can be realized on this basis.

\section{Main Elements of The Network}

To conduct the time domain simulation of the DN, the elements should be modeled. Main elements of the DN are line, load and DG. Lines generally adopt the transient model, whose three-phase form is:

$$
Z=\left[\begin{array}{lll}
Z_{a a} & Z_{a b} & Z_{a c} \\
Z_{a b} & Z_{b b} & Z_{b c} \\
Z_{a c} & Z_{b c} & Z_{c c}
\end{array}\right] \text { (1) }
$$

Loads generally adopt the constant impedance model. Provided the static load and dynamic load are considered, the transmission network processing method can be borrowed. The model of the dynamic component, DG, will be discussed in this part. 
The control strategy of grid connected DG is generally PQ control method, and PQ control requires that grid-side voltage is symmetric, here to consider grid-side voltage symmetry ( hereinafter specifically address the problem of asymmetric). In addition, DG modeling also needs to assume that the harmonic problems are ignored, and the output of the inverter is always symmetrical three-phase fundamental sinusoidal quantity.

The typical grid connected topology of the distributed power inverter is shown in Fig.1, where $L$ is filter inductor, $R$ is all inverter device loss equivalent resistance, $u_{g_{-} a b c}$ is inverter grid-side voltage. To simplify, $U_{\mathrm{dc}}$, a constant DC voltage source, is used to substitute distributed power supply side.

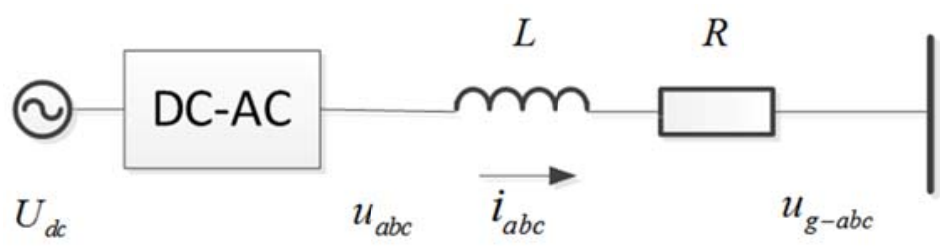

Fig.1 The grid connected topology of DG

\section{Model of distributed generation}

\section{Circuit Model of Distributed Generation}

Models of actual value:.As is shown in Fig.1, the DG circuit topology is mainly made up of DC power supplies, inverters and filters, and the inverter model has two forms: switch function model and duty model. The switch function model is generally used for electromagnetic transient simulation and accurate instantaneous value simulation, but also requires that all components are using electromagnetic model. This paper focuses on the electromechanical transient simulation of conducting RMS calculation, inverter using duty-cycle model, ignoring the state of various switches of the inverter, assuming that the inverter output voltage is symmetrical sinusoidal voltage controlled by a duty.

Assuming that system-side symmetrical three-phase voltage is:

$\left[\begin{array}{l}u_{g a} \\ u_{g b} \\ u_{g c}\end{array}\right]=\sqrt{\frac{2}{3}} U_{g}\left[\begin{array}{c}\cos \omega t \\ \cos (\omega t-2 / 3 \pi) \\ \cos (\omega t+2 / 3 \pi)\end{array}\right]$.

where $_{U_{q}}$ is the RMS of the network-side line voltage.

Assuming the inverter output voltage phase advance network side voltage of $\delta$, we have:

$\left[\begin{array}{l}u_{a} \\ u_{b} \\ u_{c}\end{array}\right]=\sqrt{\frac{2}{3}} \mathrm{KU}_{\mathrm{dc}}\left[\begin{array}{c}\cos (\omega t+\delta) \\ \cos (\omega t-2 / 3 \pi+\delta) \\ \cos (\omega t+2 / 3 \pi+\delta)\end{array}\right]$.

where $K$ is the ratio of inverter, defined as the ratio of the output line voltage and DC voltage of the inverter.

The domain circuit equation of Fig. 1 is:

$$
L \frac{d}{d t}\left[\begin{array}{l}
i_{a} \\
i_{b} \\
i_{c}
\end{array}\right]+R\left[\begin{array}{l}
i_{a} \\
i_{b} \\
i_{c}
\end{array}\right]=\left[\begin{array}{l}
u_{a} \\
u_{b} \\
u_{c}
\end{array}\right]-\left[\begin{array}{l}
u_{g a} \\
u_{g b} \\
u_{g c}
\end{array}\right] .
$$

Since the inverter control is decoupled in the dq axis, the circuit model under the dq axis can be obtained by orthogonally transforming, with transformation matrix phase angle and the network side voltage being the same: 


$$
D_{m}=\sqrt{\frac{2}{3}}\left[\begin{array}{ccc}
\cos \omega t & \cos (\omega t-2 / 3 \pi) & \cos (\omega t+2 / 3 \pi) \\
-\sin \omega t & -\sin (\omega t-2 / 3 \pi) & -\sin (\omega t+2 / 3 \pi) \\
1 / \sqrt{2} & 1 / \sqrt{2} & 1 / \sqrt{2}
\end{array}\right] .
$$

The Park's Transformation of the voltage vector:

$$
D_{m}\left[\begin{array}{l}
u_{g a} \\
u_{g b} \\
u_{g c}
\end{array}\right]=\left[\begin{array}{c}
u_{g d} \\
u_{g q} \\
0
\end{array}\right]=\left[\begin{array}{c}
U_{g} \\
0 \\
0
\end{array}\right] \quad \text { (6) } D_{m}\left[\begin{array}{c}
u_{a} \\
u_{b} \\
u_{c}
\end{array}\right]=\left[\begin{array}{c}
u_{d} \\
u_{q} \\
0
\end{array}\right]=\left[\begin{array}{c}
K U_{d c} \cos \delta \\
K U_{d c} \sin \delta \\
0
\end{array}\right] \text { (7) }
$$

Substituting (3) into (7) and (4), we have the DG's actual value equation of the circuit model:

$$
\frac{d}{d t}\left[\begin{array}{l}
i_{d} \\
i_{q}
\end{array}\right]=\left[\begin{array}{cc}
-R / L & \omega \\
-\omega & -R / L
\end{array}\right]\left[\begin{array}{l}
i_{d} \\
i_{q}
\end{array}\right]+\frac{K}{L}\left[\begin{array}{c}
\cos \delta \\
\sin \delta
\end{array}\right] u_{d c}-\frac{1}{L}\left[\begin{array}{l}
u_{g d} \\
u_{g q}
\end{array}\right] \text {. }
$$

It should be noted that according to (6), $u_{g q}=0$, and for the explanation of the principle and the writing symmetry, all $u_{g q}$ in this paper represent $u_{g q}=0$.

Models of per-unit value:Models of actual value have drawbacks that control parameter selection will have no uniform reference standard as actual system capacity, voltage changes, in addition a single-phase value and a three-phase value are not uniform, the line value with the phase value are not uniform, $\mathrm{abc}$ and $\mathrm{dq}$ coordinate values are not uniform. Thus, selecting the network-side reference value to per-unit normalize each dynamic element makes the interface processing of the dynamic element of the whole network and the network simple. The variables and parameters per-unit normalized can get rid of the limitations of the specific physical quantity, reflecting the essential mathematical characteristics of the model.

Let network-side line voltage and power base value be $U_{B}, S_{B}$, then $U_{\mathrm{ph}_{B} \mathrm{~B}}=\frac{1}{\sqrt{3}} U_{B}, I_{B}=I_{\mathrm{ph}_{-B}}=\frac{S_{B}}{\sqrt{3} U_{B}}$, $Z_{B}=\frac{U_{B}^{2}}{S_{B}}$, so DG side has $S_{\text {dq B }}=S_{B}, U_{\text {dq B }}=U_{B}$, according to $S_{\text {dq B }}=I_{\text {dq B }} U_{\text {dq_B }}$, we can deduce that $I_{\mathrm{dq} \mathrm{B}}=\sqrt{3} I_{\mathrm{B}}, Z_{\mathrm{dq} \_\mathrm{B}}=Z_{\mathrm{B}}$. Let time base value be $t_{t_{\mathrm{B}}}=\frac{1}{\omega}$. Now each value has its base value.

According to (8), we have:

$$
\frac{d}{\omega d t}\left[\begin{array}{l}
i_{d} \\
i_{q}
\end{array}\right]=\left[\begin{array}{cc}
-R / \omega L & 1 \\
-1 & -R / \omega L
\end{array}\right]\left[\begin{array}{l}
i_{d} \\
i_{q}
\end{array}\right]+\frac{K}{\omega L}\left[\begin{array}{c}
\cos \delta \\
\sin \delta
\end{array}\right] u_{d c}-\frac{1}{\omega L}\left[\begin{array}{l}
u_{g d} \\
u_{g q}
\end{array}\right] .
$$

According to the base value obtained above, per-unit normalize(9) and gain the per-unit value equation of DG's circuit model:

$$
\frac{d}{d t^{*}}\left[\begin{array}{l}
i_{d}^{*} \\
i_{q}^{*}
\end{array}\right]=\left[\begin{array}{cc}
-R^{*} / X^{*} & 1 \\
-1 & -R^{*} / X^{*}
\end{array}\right]\left[\begin{array}{l}
i_{d}^{*} \\
i_{q}^{*}
\end{array}\right]+\frac{K^{*}}{X^{*}}\left[\begin{array}{c}
\cos \delta \\
\sin \delta
\end{array}\right]-\frac{1}{X^{*}}\left[\begin{array}{l}
u_{g d}^{*} \\
u_{g q}^{*}
\end{array}\right] .(10)
$$

Where the base value of the ratio is introduced as $\mathrm{K}_{\text {base }}=\frac{U_{d c}}{U_{\text {base }}}$, making the reasonable value of $K^{*}$ is about 1 no matter how much the DC side or the network side voltages are, which makes it easier to design duty cycle limiting link. With (7), it can be inferred that $K^{*}$ and $\delta^{*}$ are related to the voltage control value or output value of the inverter under the dq axis, the relation is:

$$
\left\{\begin{array}{l}
u_{q}^{*}=K^{*} \cos \delta \\
u_{q}^{*}=K^{*} \sin \delta
\end{array}\right.
$$

The orthogonal park matrix is different. Let the voltage in the equation $D_{m}\left[\begin{array}{c}u_{a} \\ u_{b} \\ u_{c}\end{array}\right]=\left[\begin{array}{c}u_{d} \\ u_{q} \\ 0\end{array}\right]$ per-unit 
normalized and we have $\frac{1}{\sqrt{3}} D_{m}\left[\begin{array}{c}u_{a}^{*} \\ u_{b}^{*} \\ u_{c}^{*}\end{array}\right]=\left[\begin{array}{c}u_{d}^{*} \\ u_{q}^{*} \\ 0\end{array}\right]$.Similarly, we have $\frac{1}{\sqrt{3}} D_{m}\left[\begin{array}{c}i_{a}^{*} \\ i_{b}^{*} \\ i_{c}^{*}\end{array}\right]=\left[\begin{array}{c}i_{d}^{*} \\ i_{q}^{*} \\ 0\end{array}\right]$.

So the orthogonal park matrix in the form of per-unit value is:

$$
\frac{1}{\sqrt{3}} D_{m}=\frac{\sqrt{2}}{3}\left[\begin{array}{ccc}
\cos \omega t & \cos (\omega t-2 / 3 \pi) & \cos (\omega t+2 / 3 \pi) \\
-\sin \omega t & -\sin (\omega t-2 / 3 \pi) & -\sin (\omega t+2 / 3 \pi) \\
1 / \sqrt{2} & 1 / \sqrt{2} & 1 / \sqrt{2}
\end{array}\right] \text {. }
$$

Hereinafter, for convenient writing, all per-unit values' superscripts '*' are omitted, and all parameters and variables are per-unit values if there are no special instructions; the orthogonal park matrix is referred to as the park or $D_{m}$ transformation.

\section{Distributed Generation Controller Model}

The DG grid-connected in the DN usually adopts PQ control strategy, i.e. its output refers to a given power, voltage problems and imbalance problems caused by load fluctuations in grid are solved by large power grid. PQ control strategy adopts power outer loop and current inner loop, and its control mode is generally PI link.

Power outer loop. The power injected into the grid by DG under the dq axis is:

When the phase angles of transformation matrix and the network-side voltage are uniform, $u_{g q}=0$, and substituted the equation into (13), we have:

$$
S_{d g}=\left(u_{g d}+j u_{g q}\right) \times\left(i_{d}+j i_{q}\right)^{*} . \quad \text { (13) }\left\{\begin{array}{l}
P_{d g}=u_{g d} i_{d} \\
Q_{d g}=-u_{g d} i_{q}
\end{array}\right.
$$

The active and reactive outputs of the DG can be separately controlled by the control of $i_{d}$ and $i_{q}$. And this is the ideology of the power decoupling, which is based on the correctness of the (2) and (5) that the network-side voltage is symmetric and the phase angles of transformation matrix and the network-side voltage are uniform. The power control loop is shown in Fig.2.

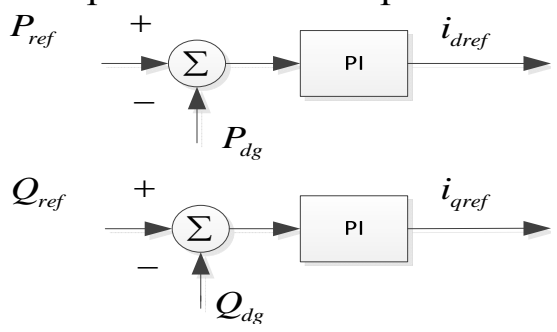

Fig2 The power outer loop control chart

Current inner loop:The reference output values, $i_{d r e f}$ and $i_{q r e f}$, of $i_{d}$ and $i_{q}$ are obtained by the power outer loop control. In order to achieve accurate current inner loop control, we need to consider the actual circuit model. Organizing (10), we have:

$$
\left\{\begin{array}{l}
u_{d}=\left(X \frac{d}{d t}+R\right) i_{d}-X i_{q}+u_{g d} \\
u_{q}=X\left(\frac{d}{d t}+R\right) i_{q}+X i_{d}+u_{g q}
\end{array} .\right.
$$

The purpose of current inner loop control is to obtain the required voltage PWM wave signal by giving the current reference and controlling the current, it is perfect if $u_{d}$ and $u_{q}$ can be controlled respectively through the control of $i_{d}$ and $i_{q}$. According to (15), we can know there is a coupling of the output voltage and the output current under the dq axis, which is manifested as the fact that $u_{d}$ is influenced by $i_{q}$ and $u_{q}$ is influenced by $i_{d}$ and they are both also affected by the network-side voltage $u_{g d}$. The current controllers commonly used adopt feedforward decoupling control strategy to achieve decoupling. When the current loop also uses PI regulator, the control equation according to 
the feedforward decoupling control strategy is:

$$
\left\{\begin{array}{l}
u_{d}=\left(k_{p}+\frac{k_{i}}{s}\right)\left(i_{d r e f}-i_{d}\right)-X i_{q}+u_{g d} \\
u_{q}=\left(k_{p}+\frac{k_{i}}{s}\right)\left(i_{q r e f}-i_{q}\right)+X i_{d}+u_{g q}
\end{array} .\right.
$$

The current inner decoupling control chart according to (16) is shown in Fig.3.

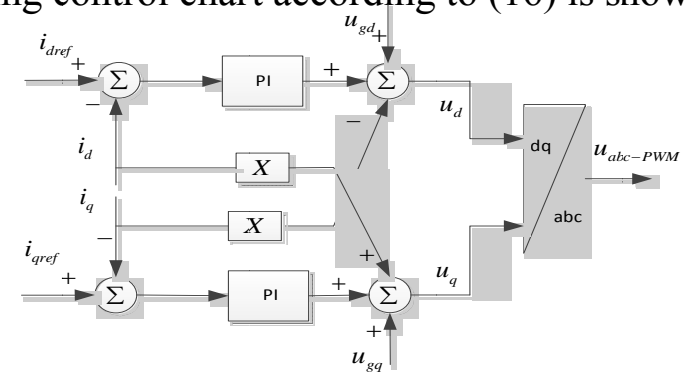

Fig3 The current inner decoupling control chart

To sum up, the complete PQ control block diagram is shown in Fig.4. It should be noted that:

- All parameters and variables should be in the form of the p.u.;

- All the PI output value and the ratio $K$ can set a limiting link;

- $R+j X$ is a filter and loss link, which model is shown in (10), and its inverter-side voltage given by the controller output;

- The precondition of the block diagram is that the network-side voltage is three-phase symmetric and the phaseangles of transformation matrix and the network-side voltage are uniform;

- The minus must be added before the PI3 link, which is due to the (14) where there is a negative correlation between $Q_{d q}$ and $i_{q}$.

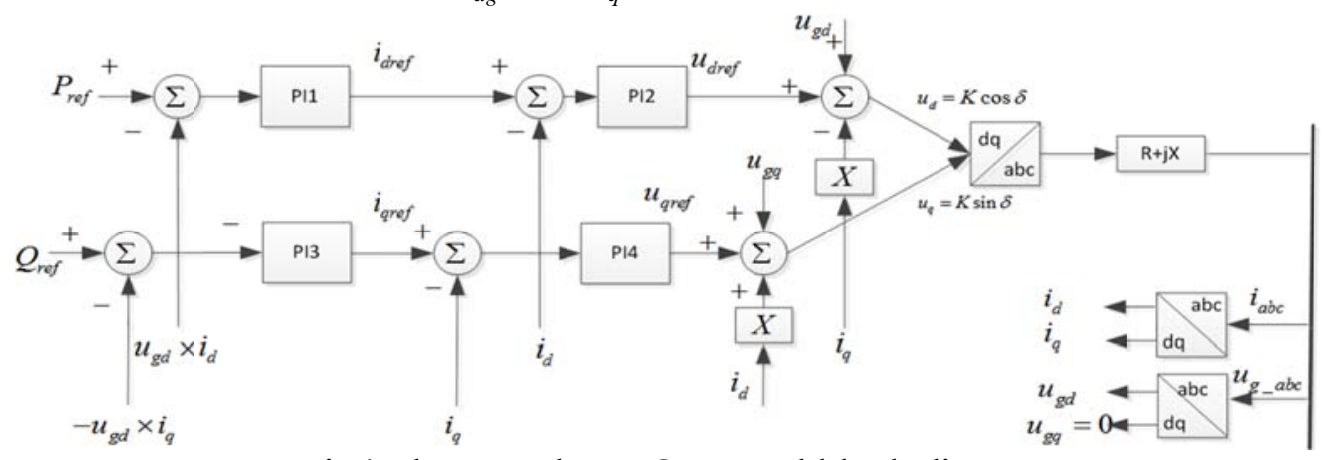

Fig4 The complete PQ control block diagram

\section{The solution to asymmetric grid-side voltageE}

Either modeling the inverter circuit model or designing the controller of DG are all based on the precondition that the network-side voltage is three-phase symmetric and the phase angles of transformation matrix and the network-side voltage are uniform, but the actual DN's asymmetric network and operation will inevitably lead to the network voltage asymmetry of each node, thus it is required to solve the contradiction between the symmetric model and the actual asymmetric network voltage. The network voltage of the actual DN's each node is asymmetric, but the voltage asymmetry is relatively low under normal circumstances, according to GB-T 15543-2008 GB provisions which stipulates that the negative sequence voltage unbalance of common connection points and load nodes in the power system can not exceed 5\%[14-16]. So grid voltage asymmetry is low when it is non-fault(otherwise this operating mode is defective), in addition DG injection current is generally also close to sinusoidal current, that is to say the current asymmetry is also low (also assuming less than 5\%), then the negative sequence and zero-sequence power only account for less than $0.25 \%$ of the injectionpower, and the majority of DG injection power is in positive sequence. If 
DG's power is given as symmetric positive-sequence, the contradiction can be solved. To conclude, the solution to the contradiction in this paper is: get the positive sequence voltage after measuring the network-side three-phase voltage, then ignore the zero-sequence and negative sequence components, and select the phase of $D_{m}$ consistent with the phase of the positive sequence voltage.

According to the DG model in this paper, we can obtain positive sequence current. Even if the network side voltage is asymmetric, only positive sequence power inject into the network because the sum of the three-phase power constituted by the positive sequence current and negative sequence, zero-sequence voltage is zero with positive sequence current injecting into the network.

\section{Conclusions}

We have accomplished modeling the DG's circuit and per-uniting the model, and modeling the controller. After that, in view of the contradiction between the symmetric DG model and the asymmetric voltage of the DN, we have proposed a solution that extracting positive sequence component, Park array tracking the phase angle of thepositive sequence component.

The drawbacks of this paper is that we have not modeled and studied the complex source side of the DG, and the output characteristics of DG are different from the reality to some extent.

\section{Acknowledgements}

This Work was supported by the National Key Technology R\&D Program of China (2013BAA02B02)

\section{References}

[1] Ioulia T. Papaioannou,ArtursPurvins,EvangelosTzimas. Demand shifting analysis at high penetration of distributed generation in low voltage grids[J]. International Journal of Electrical Power and Energy Systems,2013,44(1).

[2] RahmatallahPoudineh,ToorajJamasb. Distributed generation, storage, demand response and energy efficiency as alternatives to grid capacity enhancement[J]. Energy Policy,2013.

[3] Kristopher A. Pruitt,Robert J. Braun,Alexandra M. Newman. Evaluating shortfalls in mixed-integer programming approaches for the optimal design and dispatch of distributed generation systems[J]. Applied Energy,2013,102.

[4] Wen-Shan Tan,MohammadYusriHassan,Md Shah Majid,Hasimah Abdul Rahman. Optimal distributed renewable generation planning: A review of different approaches[J]. Renewable and Sustainable Energy Reviews,2013,18.

[5] SeyyedMostafaAbedi,Mahmoud Reza Haghifam. Second Revenue Stream for Distributed Generation in the Presence of Reliability Insurance[J]. International Journal of Electrical Power and Energy Systems, 2014.

[6] Pedro Faria,TiagoSoares,ZitaVale,HugoMorais. Distributed generation and demand response dispatch for a virtual power player energy and reserve provision[J]. Renewable Energy,2014.

[7] Robert J. Flores,Brendan P. Shaffer,JacobBrouwer. Dynamic distributed generation dispatch strategy for lowering the cost of building energy[J]. Applied Energy,2014,123.

[8] B. Asare-Bediako,W.L. Kling,P.F. Ribeiro. Future residential load profiles: Scenario-based analysis of high penetration of heavy loads and distributed generation[J]. Energy \& Buildings,2014,75.

[9] Lucian IoanDulău,MihailAbrudean,DorinBică. Effects of Distributed Generation on Electric Power Systems[J]. Procedia Technology,2014,12. 
[10] Lucian IoanDulău,MihailAbrudean,DorinBică. Distributed Generation Technologies and Optimization[J]. Procedia Technology,2014,12. 